\title{
Studies of strong and electroweak interactions using final state photon emission in hadronic $Z^{0}$ decays
}

OPAL Collaboration

P.D. Acton ${ }^{25}$, G. Alexander ${ }^{23}$, J. Allison ${ }^{16}$, P.P. Allport ${ }^{5}$, K.J. Anderson ${ }^{9}$, S. Arcelli ${ }^{2}$, A. Astbury ${ }^{28}$, D. Axen ${ }^{29}$, G. Azuelos ${ }^{18, a}$, G.A. Bahn ${ }^{16}$, J.T.M. Baines ${ }^{16}$, A.H. Ball ${ }^{17}$, J. Banks ${ }^{16}$, R.J. Barlow ${ }^{16}$, S. Barnett ${ }^{16}$, J.R. Batley ${ }^{5}$, G. Beaudoin ${ }^{18}$, A. Beck ${ }^{23}$, G.A. Beck ${ }^{13}$, J. Becker ${ }^{10}$, T. Behnke ${ }^{27}$, K.W. Bell ${ }^{20}$, G. Bella ${ }^{23}$, P. Bentkowski ${ }^{18}$, P. Berlich ${ }^{10}$, S. Bethke ${ }^{11}$, O. Biebel ${ }^{3}$, U. Binder ${ }^{10}$, I.J. Bloodworth ${ }^{1}$, P. Bock ${ }^{11}$, B. Boden ${ }^{3}$, H.M. Bosch ${ }^{11}$, H. Breuker ${ }^{8}$, P. Bright-Thomas ${ }^{25}$, R.M. Brown ${ }^{20}$, A. Buijs ${ }^{8}$, H.J. Burckhart ${ }^{8}$, C. Burgard ${ }^{27}$, P. Capiluppi ${ }^{2}$, R.K. Carnegie ${ }^{6}$, A.A. Carter ${ }^{13}$, J.R. Carter ${ }^{5}$, C.Y. Chang ${ }^{17}$, D.G. Charton ${ }^{8}$, S.L. Chu ${ }^{4}$, P.E.L. Clarke ${ }^{25}$, I. Cohen ${ }^{23}$, J.C. Clayton ${ }^{1}$, W.J. Collins ${ }^{5}$, J.E. Conboy ${ }^{15}$, M. Cooper ${ }^{22}$, M. Coupland ${ }^{14}$, M. Cuffiani ${ }^{2}$, S. Dado ${ }^{22}$, G.M. Dallavalle ${ }^{2}$, S. De Jong ${ }^{13}$, L.A. del Pozo ${ }^{5}$, H. Deng ${ }^{17}$, A. Dieckmann ${ }^{11}$, M. Dittmar ${ }^{4}$, M.S. Dixit ${ }^{7}$, E. do Couto e Silva ${ }^{12}$, J.E. Duboscq ${ }^{8}$, E. Duchovni ${ }^{26}$, G. Duckeck ${ }^{11}$, I.P. Duerdoth ${ }^{16}$, D.J.P. Dumas ${ }^{6}$, P.A. Elcombe ${ }^{5}$, P.G. Estabrooks ${ }^{6}$, E. Etzion ${ }^{23}$, H.G. Evans ${ }^{9}$, F. Fabbri' ${ }^{2}$, M. Fierro ${ }^{2}$, M. Fincke-Keeler ${ }^{28}$, H.M. Fischer ${ }^{3}$, D.G. Fong ${ }^{17}$, M. Foucher ${ }^{17}$, A. Gaidot ${ }^{21}$, O. Ganel ${ }^{26}$, J.W. Gary ${ }^{4}$, J. Gascon ${ }^{18}$, R.F. McGowan ${ }^{16}$, N.I. Geddes ${ }^{20}$, C. Geich-Gimbel ${ }^{3}$, S.W. Gensler ${ }^{9}$, F.X. Gentit ${ }^{21}$, G. Giacomelli2, R. Giacomelli' ${ }^{2}$, V. Gibson ${ }^{5}$, W.R. Gibson ${ }^{13}$, J.D. Gillies ${ }^{20}$, J. Goldberg ${ }^{22}$, M.J. Goodrick ${ }^{5}$, W. Gorn ${ }^{4}$, C. Grandi ${ }^{2}$, F.C. Grant ${ }^{5}$, J. Hagemann ${ }^{27}$, G.G. Hanson ${ }^{12}$, M. Hansroul ${ }^{8}$, C.K. Hargrove ${ }^{7}$, P.F. Harrison ${ }^{13}$, J. Hart ${ }^{8}$, P.M. Hattersley ${ }^{1}$, M. Hauschild ${ }^{8}$, C.M. Hawkes ${ }^{8}$, E. Heflin ${ }^{4}$, R.J. Hemingway ${ }^{6}$, R.D. Heuer ${ }^{8}$, J.C. Hill ${ }^{5}$, S.J. Hillier ${ }^{8}$, T. Hilse ${ }^{10}$, D.A. Hinshaw ${ }^{18}$, J.D. Hobbs ${ }^{8}$, P.R. Hobson ${ }^{25}$, D. Hochman ${ }^{26}$, R.J. Homer ${ }^{1}$, A.K. Honma ${ }^{28, a}$, R.E. Hughes-Jones ${ }^{16}$, R. Humbert ${ }^{10}$, P. Igo-Kemenes ${ }^{11}$, H. Ihssen ${ }^{11}$, D.C. Imrie ${ }^{25}$, A.C. Janissen ${ }^{6}$, A. Jawahery ${ }^{17}$, P.W. Jeffreys ${ }^{20}$, H. Jeremie ${ }^{18}$, M. Jimack ${ }^{2}$, M. Jobes ${ }^{1}$, R.W.L. Jones ${ }^{13}$, P. Jovanovic ${ }^{1}$, C. Jui ${ }^{4}$, D. Karlen ${ }^{6}$, K. Kawagoe ${ }^{24}$, T. Kawamoto ${ }^{24}$, R.K. Keeler ${ }^{28}$, R.G. Kellogg ${ }^{17}$, B.W. Kennedy ${ }^{15}$, S. Kluth ${ }^{5}$, T. Kobayashi ${ }^{24}$, D.S. Koetke ${ }^{8}$, T.P. Kokott ${ }^{3}$, S. Komamiya ${ }^{24}$, L. Köpke ${ }^{8}$, J.F. Kral ${ }^{8}$, R. Kowalewski ${ }^{6}$, J. von Krogh ${ }^{11}$, J. Krol1 ${ }^{9}$, M. Kuwano ${ }^{24}$, P. Kyberd ${ }^{13}$, G.D. Lafferty ${ }^{16}$, R. Lahmann ${ }^{17}$, F. Lamarche ${ }^{18}$, J.G. Layter ${ }^{4}$, P. Leblanc ${ }^{18}$, A.M. Lee ${ }^{17}$, M.H. Lehto ${ }^{15}$, D. Lellouch ${ }^{26}$, C. Leroy ${ }^{18}$, J. Letts ${ }^{4}$, S. Levegrün ${ }^{3}$, L. levinson ${ }^{26}$, S.L. Lloyd ${ }^{13}$, F.K. Loebinger ${ }^{16}$, J.M. Lorah ${ }^{17}$, B. Lorazo ${ }^{18}$, M.J. Losty ${ }^{7}$, X.C. Lou ${ }^{12}$, J. Ludwig ${ }^{10}$, M. Mannelli ${ }^{8}$, S. Marcellini ${ }^{2}$, G. Maringer ${ }^{3}$, C. Markus ${ }^{3}$, A.J. Martin ${ }^{13}$, J.P. Martin ${ }^{18}$, T. Mashimo ${ }^{24}$, P. Mättig ${ }^{3}$, U. Maur ${ }^{3}$, J. McKenna ${ }^{28}$, T.J. McMahon ${ }^{1}$, J.R. McNutt ${ }^{25}$, F. Meijers ${ }^{8}$, D. Menszner ${ }^{11}$, F.S. Merritt ${ }^{9}$, H. Mes ${ }^{7}$, A. Michelini ${ }^{8}$, R.P.Middleton ${ }^{20}$, G. Mikenberg ${ }^{26}$, J. Mildenberger ${ }^{6}$, D.J. Miller ${ }^{15}$, R. Mir ${ }^{12}$, W. Mohr ${ }^{10}$, C. Moisan ${ }^{18}$, A. Montanari ${ }^{2}$, T. Mori ${ }^{24}$, M. Morii ${ }^{24}$, T. Mouthuy ${ }^{12, \text { b }, ~ B . ~ N e l l e n ~}{ }^{3}$, H.H. Nguyen ${ }^{9}$, M. Nozaki ${ }^{24}$, S.W. O’Neale ${ }^{1}$, F.G. Oakham ${ }^{7}$, F. Odorici ${ }^{2}$, H.O. Ogren ${ }^{12}$, C.J. Oram ${ }^{28, a}$, M.J. Oreglia ${ }^{9}$, S. Orito ${ }^{24}$, J.P. Pansart ${ }^{21}$, B. Panzer-Steindel ${ }^{8}$, P. Paschievici ${ }^{26}$, G.N. Patrick ${ }^{20}$, N. Paz-Jaoshvili ${ }^{23}$, P. Pfister ${ }^{10}$, J.E. Pilcher ${ }^{9}$, J. Pinfold ${ }^{31}$, D. Pitman ${ }^{28}$, D.E. Plane ${ }^{8}$, P. Poffenberger ${ }^{28}$, B. Poli ${ }^{2}$, A. Pouladdej ${ }^{6}$, T.W. Pritchard ${ }^{13}$, H. Przysiezniak ${ }^{18}$, G. Quast ${ }^{27}$, M.W. Redmond ${ }^{9}$, D.L. Rees ${ }^{8}$, G.E. Richards ${ }^{16}$, D. Robinson ${ }^{8}$, A. Rollnik ${ }^{3}$, J.M. Roney ${ }^{28, c}$, E. Ros ${ }^{8}$, S. Rossberg ${ }^{10}$, A.M. Rossi ${ }^{2}$, M. Rosvick ${ }^{28}$, P. Routenburg ${ }^{6}$, K. Runge $^{10}$, O. Runolfsson ${ }^{8}$, D.R. Rust ${ }^{12}$, M. Sasaki ${ }^{24}$, C. Sbarra ${ }^{8}$, A.D. Schaile ${ }^{10}$, O. Schaile ${ }^{10}$, W. Schappert ${ }^{6}$, P. Scharff-Hansen ${ }^{8}$, P. Schenk ${ }^{4}$, B. Schmitt ${ }^{3}$, H. von der Schmitt ${ }^{11}$, S. Schreiber ${ }^{3}$, C. Schwick ${ }^{27}$, J. Schwiening ${ }^{3}$, W.G. Scott ${ }^{20}$, M. Settles ${ }^{12}$, T.G. Shears ${ }^{5}$, B.C. Shen ${ }^{4}$, C.H. Shepherd-Themistocleous ${ }^{7}$, P. Sherwood ${ }^{15}$, R. Shypit ${ }^{29}$, A. Simon ${ }^{3}$, P. Singh ${ }^{13}$, G.P. Siroli ${ }^{2}$, A. Skuja ${ }^{17}$, A.M. Smith ${ }^{8}$, T.J. Smith ${ }^{28}$, G.A. Snow ${ }^{17}$, R. Sobie ${ }^{28, c}$, R.W. Springer ${ }^{17}$, M. Sproston ${ }^{20}$, K. Stephens ${ }^{16}$, J. Steuerer ${ }^{28}$, R. Ströhmer ${ }^{11}$, D. Strom ${ }^{30}$, T. Takeshita ${ }^{24, d}$, P. Taras $^{18}$, S. Tarem ${ }^{26}$, M. Tecchio ${ }^{9}$, P. Teixeira-Dias ${ }^{11}$, N. Tesch ${ }^{3}$, N.J. Thackray ${ }^{1}$, M.A. Thomson ${ }^{15}$, E. Torrente-Lujan ${ }^{22}$, G. Transtromer ${ }^{25}$, N.J. Tresilian ${ }^{16}$, T. Tsukamoto ${ }^{24}$, M.F. Turner ${ }^{8}$, G. Tysarczyk-Niemeyer ${ }^{11}$, D. Van den plas $^{18}$, R. Van Kooten ${ }^{27}$, G.J. VanDalen, G. Vasseur ${ }^{21}$, C.J. Virtue ${ }^{7}$, A. Wagner ${ }^{27}$, D.L. Wagner ${ }^{9}$, C. Wahl ${ }^{10}$, J.P. Walker ${ }^{1}$, C.P. Ward ${ }^{5}$, D.R. Ward ${ }^{5}$, P.M. Watkins ${ }^{1}$, A.T. Watson 1 , N.K. Watson ${ }^{8}$, M. Weber ${ }^{11}$, P. Weber ${ }^{6}$, P.S. Wells ${ }^{8}$, N. Wermes ${ }^{3}$, M.A. Whalley ${ }^{1}$, G.W. Wilson ${ }^{4}$, J.A. Wilson ${ }^{1}$, V.-H. Winterer ${ }^{10}$, T. Wlodek ${ }^{26}$, S. Wotton ${ }^{11}$, T.R. Wyatt ${ }^{16}$, R. Yaari ${ }^{26}$, A. Yeaman ${ }^{13}$, G. Yekutieli' ${ }^{26}$, M. Yurko ${ }^{18}$, W. Zeuner ${ }^{8}$, G.T. Zorn ${ }^{17}$

\footnotetext{
' School of Physics and Space Research, University of Birmingham, Birmingham B152TT, UK

${ }^{2}$ Dipartimento di Fisica dell' Università di Bolgona and INFN, Bologna, 40126, Italy

${ }^{3}$ Physikalisches Institut, Universität Bonn, W-5300 Bonn 1, Germany

${ }^{4}$ Department of Physics, University of California, Riverside, CA 92521 USA

${ }^{5}$ Cavendish Laboratory, Cambridge CB3 0HE, UK

${ }^{6}$ Carleton University, Department of Physics, Colonel By Drive, Ottawa, Ontario K1S5B6, Canada

${ }^{7}$ Centre for Research in Particle Physics, Carleton University, Ottawa, Ontario K 1S 5B6, Canada

${ }^{8}$ CERN, European Organisation for Particle Physics, CH-1211 Geneva 23, Switzerland

${ }^{9}$ Enrico Fermi Institute and Department of Physics, University of Chicago, Chicago IL 60637, USA
} 
${ }^{10}$ Fakultät für Physik, Albert Ludwigs Universität, W-7800 Freiburg, Germany

${ }^{11}$ Physikalisches Institut, Universität Heidelberg, W-6900 Heidelberg, Germany

${ }^{12}$ Indiana University, Department of Physics, Swain Hall West 117, Bloomington, IN 47405, USA

${ }^{13}$ Queen Mary and Westfield College, University of London, London E1 4NS, UK

${ }^{14}$ Birkbeck College, London WC1E 7HV, UK

${ }^{15}$ University College London, London WC1E6BT, UK

${ }^{16}$ Department of Physics, Schuster Laboratory, The University, Manchester M13 9PL, UK

${ }^{17}$ Department of Physics, University of Maryland, College Park, MD 20742, USA

${ }^{18}$ Laboratoire de Physique Nucléaire, Université de Montréal, Montréal, Quebec, H3C 3J7, Canada

${ }^{20}$ Rutherford Appleton Laboratory, Chilton, Didcot, Oxfordshire OX110QX, UK

${ }^{21}$ DAPNIA/SPP, Sacley, F-91191 Gif-sur-Yvette, France

${ }^{22}$ Department of Physics, Technicon-Israel Institute of Technology, Haifa 32000, Israel

${ }^{23}$ Department of Physics and Astronomy, Tel Aviv University, Tel Aviv 69978, Israel

${ }^{24}$ International Centre for Elementary Particle Physics and Department of Physics, University of Tokyo, Tokyo 113, and Kobe

University, Kobe 657, Japan

${ }^{25}$ Brunel University, Uxbridge, Middlesex UB8 3PH UK

${ }^{26}$ Nuclear Physics Department, Weizmann Institute of Science, Rehovot, 76100, Israel

${ }^{27}$ Universität Hamburg/DESY, II Institut für Experimental Physik, W-2000 Hamburg 52, Germany

${ }^{28}$ University of Victoria, Department of Physics, P O Box 3055, Victoria BC V8W 3P6, Canada

${ }^{29}$ University of British Columbia, Department of Physics, 6224 Agriculture Road, Vancouver BC V6T 1Z1, Canada

${ }^{30}$ University of Oregon, Department of Physics, Eugene, OR 97403 , USA

${ }^{31}$ University of Alberta, Department of Physics, Edmonton AB T6G 2J1, Canada

Received 27 January 1993

\begin{abstract}
The cross section and jet rates of $Z^{0}$ decays into photons and quarks are compared with matrix element Monte Carlos of $\mathscr{O}\left(\alpha \alpha_{s}\right)$. Good agreement is found between data and theoretical predictions. From the ratio of events with three jets plus a photon to those with two jets plus a photon, $\alpha_{\mathrm{s}}$ in first order is determined to be $0.176 \pm 0.010$. Combining the cross section of final state photon events with the LEP average hadronic partial decay width of the $Z^{0}$, the widths of the $Z^{0}$ into up and down type quarks can be calculated. The results

$\Gamma_{u \text {-type }}=242 \pm 46 \mathrm{MeV}$;

$\Gamma_{d \text {-type }}=419 \pm 30 \mathrm{MeV}$

are in good agreement with the standard model expectation. A comparison of the measured cross section as a function of $y_{\text {cut }}$ with predictions of QCD shower models shows that, at the current level of accuracy ARIADNE and, to a lesser extent, HERWIG and JETSET can reproduce the measurement.
\end{abstract}

\section{Introduction}

Photon emission from quarks $[1,2]$ is a reference process which may be compared with gluon emission in studies of the strong interaction. As shown in [3-5], measurements of the yield and properties of photons are testing grounds of QCD shower models that give a good account of inclusive multihadronic events in $e^{+} e^{-}$collisions. In

a Also at TRIUMF, Vancouver, Canada V6T 2A3

b Now at Centre de Physique des Particules de Marseille, Faculté des Sciences de Luminy, Marseille

c And IPP, University of Victoria, Department of Physics, PO Box 3055, Victoria BC V8W 3P6, Canada

d Also at Shinshu University, Matsumoto 390, Japan our previous publications $[3,4]$ we also made a first comparison of data with a matrix element calculation [6] of $\mathscr{O}\left(\alpha \alpha_{\mathrm{s}}\right)$. However, this comparison was limited since it was impossible to apply identical definitions for the final state photon events in the data and the theoretical calculation. With the publication of Monte Carlo simulations [7-9] based on matrix element calculations, which generate the four vectors of the quarks, the gluon and the photon, this obstacle has been overcome and a detailed comparison of data and calculation becomes possible. Apart from allowing various QCD tests, the matrix element calculations provide a basis for the determination of the electroweak quark couplings [10] and for the search for rare or non-standard $Z^{0}$ decays [11].

A comparison of data and the predictions of matrix element Monte Carlos for the cross section and jet rates will be presented in this letter. The data were recorded during 1990 and 1991 with the OPAL detector at the LEP $e^{+} e^{-}$collider. In comparison with our previous publications [3,4] this represents an almost threefold increase of final state photon events. We use this larger data sample, together with the improved theoretical calculation of the expected yield, to update our measurement of the electroweak couplings of up and down type quarks. We also make a more sensitive comparison with the QCD shower models JETSET [12], ARIADNE [13] and HERWIG $[14,15]$.

The letter starts with a summary of the features of the OPAL detector pertinent to this analysis. We describe the event selection in Sect. 3. The background to our final state photon sample and the acceptance of the photon selection will be discussed in Sect. 4. We introduce the matrix element Monte Carlo in Sect. 5. We present in Sect. 6 our measurement of the total photon yield and of the jet multiplicities associated with photon emission and determine $\alpha_{\mathrm{s}}$. Finally, we give in Sects. 7 and 8 updates of our measurement of the electroweak quark couplings and of the comparison to QCD shower models. 


\section{The OPAL detector}

The analysis is based on an integrated luminosity of approximately $21 \mathrm{pb}^{-1}$ collected with the OPAL detector [16] at LEP. The data were recorded around the $Z^{0}$ pole at centre-of-mass energies $E_{\mathrm{cm}}$ between 88.28 and $94.28 \mathrm{GeV}$.

The most important components of OPAL for this study are the tracking chambers and the electromagnetic calorimeter. The central detector provides a measurement of the momenta of charged particles in a magnetic field of $0.435 \mathrm{~T}$. The barrel part of the electromagnetic calorimeter covers the complete azimuthal range up to polar angles satisfying $|\cos \theta|<0.82$, where $\theta$ is defined relative to the direction of the incoming electrons. It consists of 9440 lead glass blocks of 24.6 radiation lengths, pointing towards the interaction region and each subtending an angular region of approximately $40 \times 40 \mathrm{mrad}^{2}$. The two endcap calorimeters consist together of 2264 lead glass blocks and cover the region of $0.82 \leqq|\cos \theta| \leqq 0.98$.

\section{The event selection}

For this analysis we combine the event samples collected in 1990 and 1991. The results from the 1990 data, corrected for acceptance and efficiency have been published previously $[3,4]$ and are unchanged except for a re-evaluation of the hadronic background. The analysis approach for the 1991 data is the same as that used for 1990 , details of which can be found elsewhere $[3,4]$. For the calculation of efficiencies and the evaluation of the systematic uncertainties we use data reference samples from the corresponding years. In the following we describe the event selection and the procedure for correcting the data, for the data sample collected in 1991. At the end of the next section we summarise the most important differences in the treatment of the data from 1990 and 1991.

The selection of events for this analysis proceeds in two stages: first we select multihadronic $Z^{0}$ decays and in a second step we search for isolated, high energy photon candidates.

Multihadronic events are required [17] to have at least five well measured tracks and more than seven clusters in the electromagnetic calorimeter. A well measured track, reconstructed from at least 20 hits in the tracking chambers, must have a minimum momentum transverse to the beam direction of $50 \mathrm{MeV}$, a reconstructed distance of closest approach to the beam axis of less than $2 \mathrm{~cm}$, and a longitudinal displacement along the beam direction from the nominal interaction point of less than $40 \mathrm{~cm}$ at the point of closest approach to the beam. A cluster in the lead glass calorimeter consists of at least one block and a total energy of more than $100 \mathrm{MeV}$ in the barrel region $(|\cos \theta|<0.82)$ and of at least two adjacent blocks with a minimum total energy of $200 \mathrm{MeV}$ in the endcap region $(0.82 \leqq|\cos \theta| \leqq 0.98)$. The energy sum of all accepted clusters $\Sigma E_{\text {clu }}$ must exceed $0.1 \cdot E_{\mathrm{cm}}$. The energy deposition must be balanced along the beam direction so that $\left|\Sigma\left(E_{\mathrm{clu}} \cdot \cos \theta\right)\right| / E_{\mathrm{cm}}<0.65$. These requirements are satisfied by 353160 events. From Monte Carlo studies the acceptance for multihadronic events is estimated to be 0.984 with a systematic uncertainty of 0.004 . The fraction of background from $\tau$ pairs and two-photon processes is estimated to be less than 0.003 .

In these multihadronic $Z^{0}$ decays we search for photon candidates based on geometrical and topological criteria. To suppress background from hadrons that fake genuine photons we select high energy isolated clusters, which rarely originate from fragmentation. These clusters are required to fulfil the following criteria:

- The photon candidate has to be within a fiducial volume $(|\cos \theta|<0.72)$ of the lead glass barrel calorimeter. - The photon energy has to be larger than $7.5 \mathrm{GeV}$.

- No well measured track with a transverse momentum of more than $250 \mathrm{MeV}$ or additional cluster with an energy exceeding $250 \mathrm{MeV}$ is allowed within an isolation cone of half-angle 15 degrees around the flight direction of the photon candidate.

- The event is retained if the photon is found to lie outside a jet. In a first step the photon candidate is not considered and jets are formed from the remaining tracks and clusters. Jets are constructed by iteratively combining the pair of particles or combinations of particles that have the minimum

$y_{i j}=\frac{M_{i j}^{2}}{E_{\mathrm{vis}}^{2}}$

in each iteration. This combination process is terminated when all possible combinations give a $y_{i j}$ larger than a specified $y_{\text {cut }}$. For energies $E_{i}, E_{j}$ and the opening angle $\alpha_{i j}$ of these pairs, the quantity $M_{i j}$ is calculated using both the JADE $E 0$ scheme [18] with $M_{i j}^{2}=$ $2 E_{i} E_{j}\left(1-\cos \alpha_{i j}\right)$ and the Durham scheme [19] which defines $M_{i j}^{2}=2 \min \left(E_{i}^{2}, E_{j}^{2}\right)\left(1-\cos \alpha_{i j}\right)$. The latter recombination scheme has a better convergence of the QCD perturbative expansion and leads to an assignment of particles to jets which agrees more with intuition. The use of two different recombination schemes provides an estimate of the sensitivity to the reconstruction of partons from hadron jets. The visible energy $E_{\text {vis }}$ is calculated from all tracks and clusters including the photon. In a second step we calculate $y_{\gamma \text {,jet }}$ by pairing the photon candidate with each jet. At this stage we correct the jet energies and $E_{\text {vis }}$ for double counting of tracks and clusters from the same particle with the algorithm described in [20]. This correction has been checked with inclusive multihadronic events, with a polar angle of the thrust axis of $\left|\cos \theta_{\text {thrust }}\right|<0.80$, to ensure full containment of the events within the acceptance of the detector. Calculating $E_{\text {vis }}$ with these events, using this algorithm, we find $\left\langle E_{\mathrm{vis}}\right\rangle=a \cdot E_{\mathrm{cm}}$ with $a=0.998$. We retain an event if $y_{\gamma \text {,jet }}$ of the photon with each jet is larger than $y_{\text {cut }}$.

To suppress further background from hadrons, we demand that the cluster properties are those expected from a single photon. We select clusters based on the number of blocks, the energy weighted width and a cluster shape variable, indicating the goodness of the fit to the photon hypothesis (for details see [3]). These requirements are 
Table 1. Photon candidates and background contributions in the combined 1990 and 1991 data sample as obtained with the $E 0$ scheme. The observed yield of photon candidates $\left(N_{\text {cand }}\right)$, the background from hadrons $\left(N_{\text {had }}\right)$ and initial state radiation $\left(N_{\text {ISR }}\right)$, and the number of final state photons $\left(N_{\mathrm{FSR}}\right)$ in our sample

\begin{tabular}{lllll}
\hline$y_{\text {cut }}$ & $N_{\text {cand }}$ & \multicolumn{1}{l}{$N_{\text {had }}$} & \multicolumn{1}{l}{$N_{\text {ISR }}$} & \multicolumn{1}{l}{$N_{\text {FSR }}$} \\
\hline 0.005 & 964 & $72.9 \pm 28.6$ & $42.7 \pm 3.0$ & $848.4 \pm 42.3$ \\
0.010 & 903 & $62.1 \pm 24.5$ & $43.0 \pm 3.1$ & $797.9 \pm 38.9$ \\
0.020 & 828 & $50.5 \pm 20.0$ & $40.7 \pm 3.0$ & $736.8 \pm 35.2$ \\
0.040 & 663 & $44.7 \pm 17.6$ & $36.5 \pm 2.7$ & $581.8 \pm 31.3$ \\
0.060 & 541 & $34.1 \pm 13.5$ & $30.6 \pm 2.5$ & $476.3 \pm 27.0$ \\
0.080 & 440 & $27.9 \pm 11.1$ & $24.8 \pm 2.2$ & $387.3 \pm 23.8$ \\
0.100 & 358 & $23.3 \pm 9.6$ & $18.7 \pm 1.8$ & $316.0 \pm 21.3$ \\
0.120 & 302 & $19.2 \pm 8.1$ & $13.7 \pm 2.5$ & $269.1 \pm 19.3$ \\
0.140 & 270 & $17.9 \pm 7.7$ & $12.2 \pm 2.1$ & $239.9 \pm 18.3$ \\
0.160 & 236 & $15.8 \pm 6.4$ & $10.7 \pm 1.9$ & $209.5 \pm 16.8$ \\
0.180 & 222 & $14.0 \pm 7.2$ & $9.6 \pm 1.8$ & $198.4 \pm 16.6$ \\
0.200 & 216 & $12.2 \pm 7.0$ & $9.6 \pm 1.8$ & $194.2 \pm 16.4$ \\
\hline
\end{tabular}

unchanged from the previous publications. For the $E 0$ scheme the number of photon candidates retained for various values of $y_{\text {cut }}$ are listed in Table 1 .

\section{Background and acceptance correction}

Isospin symmetry is invoked to estimate the background rate of neutral hadrons using charged hadrons. As detailed in [3], the hadronic background contributions, mainly due to $\pi^{0} \rightarrow \gamma \gamma$ decays, are investigated using a control sample $S$ of well measured charged particles that fulfil the same energy and isolation criteria as the photon candidates. Using isospin symmetry and taking into account corrections such as the $\pi^{0}$ rejection efficiency, this translates into a background contribution from single isolated neutral particles. Contributions to $S$ from unresolvable pairs of a charged and a neutral particle were estimated from a sample $D$ of very close by tracks which together fulfil the isolation criteria discussed above. This sample $D$ is also used to estimate the number of unresolved pairs of neutral particles in our sample of photon candidates. These amount to about $7 \%$ of the total hadronic background at low $y_{\text {cut }}$ and to about $40 \%$ at high $y_{\text {cut }}$. Due to the uncertainties in the fraction of charged pions in the stable particle yield and the ratio of neutral to charged pions, we estimate an error of $40 \%$ for the background calculated from single charged tracks. A systematic error of $100 \%$ has been assigned to the contribution from unresolvable pairs of particles. The estimated hadronic background is given in the third column of Table 1.

The background from initial state radiation is estimated using the first order QED calculation of [21] as implemented in JETSET [12] taking into account the distribution of the centre-of-mass energy of the data. Depending on the value of $y_{\text {cut }}$ we estimate a background of $3.9-5.7 \%$ as given in the fourth column of Table 1 with an error due to Monte Carlo statistics.

The measured yield is corrected for acceptance and detector effects. We split the correction $c=c_{1} \cdot c_{2}=\frac{N_{p}}{N_{h}} \cdot \frac{N_{h}}{N_{\text {det }}}$

into two parts. Here $N_{p}$ denotes the number of simulated events found at the parton level with only the $y_{\text {cut }}$ requirement imposed, $N_{h}$ the number at the hadron level to which the energy and isolation requirement have also been applied and $N_{\text {det }}$ denotes the number of simulated events accepted at the detector level. The correction $c_{2}$ accounts for all effects caused by the detector and event reconstruction. The correction $c_{1}$ accounts for the isolation and energy requirement and for the fragmentation of partons (see below).

The detector correction $c_{2}$ takes into account the efficiency of photon identification, the interaction of particles in the detector, the determination of jet energies, and the geometrical acceptance. These corrections are obtained from the data and from a detailed simulation of the response of the OPAL detector [22] to hadronic events generated with JETSET [12]. As discussed in detail in [4], a shift in the reconstructed energy of jets would introduce a bias in the corrected number of photon events. To estimate whether or not the potential bias is well reproduced in the simulation we compare the energy reconstruction of jets in the data and the simulation. We define

$\delta E=\frac{E_{\text {xecon }}-E_{\text {meas }}}{E_{\text {recon }}}$,

with $E_{\text {recon }}$ the jet energy reconstructed for events with two jets and a photon from the angles between jets using massless parton kinematics, and $E_{\text {meas }}$ is the jet energy fully corrected as described in Sect. 3 and in [4]. We found the difference of the average values $\langle\delta E\rangle_{\text {data }}$ $-\langle\delta E\rangle_{\text {simulation }}$, to be $+0.016 \pm 0.020$. Allowing a global energy correction of $1.6 \%$ leads to a change of the calculated cross section of up to $1 \%$ depending on the value of $y_{\text {cut }}$. This potential change will be assigned as a systematic uncertainty.

The efficiency for photon identification is determined with a sample of 661 genuine photons from radiative lepton and $e^{+} e^{-} \rightarrow \gamma \gamma$ events. The photon efficiency, after the requirements on the cluster shape, is found to be $93.6 \pm 1.3 \%$. No energy or angular dependence is observed. We estimate the conversion probability before and inside the tracking chambers based on our knowledge of the material in the OPAL detector, including the material from the new silicon microvertex detector and the additional beam pipe [23]. We estimate the minimum conversion probability (perpendicular incidents) to be $6.3 \pm 1.2 \%$.

Combining these corrections and including an extrapolation to polar angles of more than $|\cos \theta|=0.72$, which amounts to $43 \%$, the total correction $c_{2}$ is typically 1.7 . Including the statistical error from the Monte Carlo simulation of $1 \%-2.5 \%$, the total error of $c_{2}$ is typically $3 \%$.

To compare the measurement to the matrix element calculations which predict the rates of at most three partons and a photon, we apply corrections for the energy cut and for the photon isolation requirement, which is 
related to fragmentation effects. These corrections $c_{1}$ are obtained from the JETSET shower model. The model parameters are fixed to the values optimised to describe OPAL data [24]. It was verified that the corrections do not depend significantly on these parameter values. Due to both the energy and isolation requirement the correction is large at low values of $y_{\text {cut }}$ (at $y_{\text {cut }}=0.005$ in the $E 0$ scheme $c_{1}=2.03$ ), the losses for $y_{\text {cut }}>0.06$ are small and almost exclusively due to the isolation requirement. The correction is rather constant with a value of about 1.08 between $y_{\text {cut }}$ values of 0.06 and 0.16 . It increases again slightly at high values of $y_{\text {cut }}\left(c_{1}=1.13\right.$ at $y_{\text {cut }}=0.2$ ).

For some of the studies below we also consider differential distributions:

$$
\begin{aligned}
& D_{\mathrm{FSR}}\left(y_{\mathrm{cut}}\right) \\
& =\frac{1000}{N_{M H} \delta}\left[N_{\mathrm{FSR}}\left(y_{\text {cut }}-\delta / 2\right)-N_{\mathrm{FSR}}\left(y_{\text {cut }}+\delta / 2\right)\right] \\
& =\frac{1000}{N_{M H} \delta}\left[\sum_{i} N^{i \rightarrow \text { reject }}-\sum_{i} N^{\text {reject } \rightarrow i}\right] .
\end{aligned}
$$

Here $N_{\text {FSR }}$ is the number of final state photons, $N_{M H}$ is the number of hadronic $Z^{0}$ decays and $N^{i \rightarrow \text { reject }}$ denotes the losses due to the increase of $y_{\text {cut }}$, i.e. the number of events that have a jet multiplicity $i$ at $y_{\text {cut }}-\delta / 2$ but that are rejected at $y_{\text {cut }}+\delta / 2$ because they no longer satisfy the condition $y_{\gamma, \text { jet }}>y_{\text {cut }}$ for all jets. The gains $N^{\text {reject } \rightarrow i}$ are due to events that are rejected at $y_{\text {cut }}-\delta / 2$ but are retained at $y_{\text {cut }}+\delta / 2$ with a jet multiplicity $i$. Here the corrected distributions are obtained from the observed ones by applying differential corrections factors. The losses are

$$
N^{i \rightarrow \text { reject }}=c^{i \rightarrow \text { reject }} N_{\mathrm{obs}}^{i \rightarrow \text { reject }},
$$

with the index 'obs' referring to the observed number of events. The differential correction factor is

$$
\begin{aligned}
c^{i \rightarrow \mathrm{reject}} & =c_{1}^{i \rightarrow \mathrm{reject}} \cdot c_{2}^{i \rightarrow \mathrm{reject}} \\
& =\frac{N_{p}^{i \rightarrow \mathrm{reject}}}{N_{h}^{i \rightarrow \mathrm{reject}}} \frac{N_{h}^{i \rightarrow \mathrm{reject}}}{N_{\mathrm{det}}^{i \rightarrow \mathrm{reject}}} .
\end{aligned}
$$

For the events gained by the increase of $y_{\text {cut }}, N^{\text {reject } \rightarrow i}$, the corrections have been calculated in an analogous way.

To estimate the systematic uncertainties of the integral and differential corrections we use various QCD shower models and the data. We observe the following results.

- A potential model dependence of the corrections is studied by comparing JETSET with ARIADNE [13] and HERWIG [14]. The three models use different parton showering and fragmentation schemes. Comparing the integral $c_{1}$ values from the different models we find them to agree with the reference JETSET value within $5 \%$ up to $y_{\text {cut }}=0.16$. For larger $y_{\text {cut }}$ HERWIG gives somewhat smaller $c_{1}$.

For the differential corrections we observe differences between models of about $25 \%$ at small $y_{\text {cut }}$. For $y_{\text {cut }}>0.02$ the correction factors agree within $5 \%$.
The results obtained for the integral cross section of photon events with the integral and differential correction factors from JETSET agree within $2 \%$. However, using the differential corrections from other models, we observe differences between $5 \%$ and $10 \%$ for $y_{\text {cut }}<0.02$.

- We compare, for the $E 0$ scheme, the angular distance between the nearest track or cluster and the photon candidate for both the data and the simulation and find good agreement, suggesting that the particle and energy flows are understood.

In addition we repeat the entire analysis for different isolation criteria, varying the isolation angle between 10 and 20 degrees and allowing up to $0.5 \mathrm{GeV}$ in the isolation cone. As an example, for a $y_{\text {cut }}$ value of 0.06 the corrected number of final state photons changes by $+1 \%$ for a cone of 10 degrees and by $-4.9 \%$ for a cone of 20 degrees.

As a result of these considerations, and since, at least in part, these estimates are not independent we assign the systematic error on $c_{1}$ to be the maximum of (a) either $25 \%$ of the losses due to the energy and isolation requirement, (b) the difference between the corrections from different QCD shower models, or (c) at least 5\%. Comparing these errors we find that (a) defines the error for $y_{\text {cut }}<0.04$, (c) for $0.04<y_{\text {cut }}<0.16$ and (b) for $y_{\text {cut }}>0.16$. Similar corrections are found for the Durham scheme. Here the correction factors from the different models agree within $5 \%$ for $0.004<y_{\text {cut }}<0.06$.

The main differences in the treatment of the data from 1990 [3] and that described above for 1991 are as follows:

The requirements for selecting multihadronic $Z^{0}$ decays were slightly different in 1990 , resulting in 145095 events with an acceptance of $0.975 \pm 0.010$. In 1990 the maximum energy of electromagnetic clusters allowed in the isolation cone of the photon candidate was $100 \mathrm{MeV}$. The increase of the accepted energy was necessary because of a modification in the simulation of hadronic showers, leading to a poorer description of clusters of less than $250 \mathrm{MeV}$ of hadronic origin. In 1991 a better parametrisation of the material in the OPAL detector led to an improved understanding of the energy scale of hadronic jets, resulting in a reduction of the systematic error from this source. More detailed studies of the event sample containing isolated charged particles led to an increase in the estimated background by a factor 2 to 3 . We corrected our 1990 results for this change. Our previous analysis was based on the $E 0$ scheme for the jet finding. The Durham jet finding algorithm is used as a cross check and has been applied to the data from 1991 only.

Applying all these corrections and taking into account the uncorrelated systematic uncertainties, we obtain consistent results for the data of 1990 and 1991. Typically the total cross section of final state photon events from the 1991 data is one standard deviation higher than that of 1990. For the following comparison with theoretical predictions we combine the measurements from 1990 and 1991, which corresponds to a total sample of 498255 multihadronic events. The results are shown in Table 2 and in Fig. 1. In combining the measurements we treated the errors of $c_{1}$, of the background and of the jet recon- 

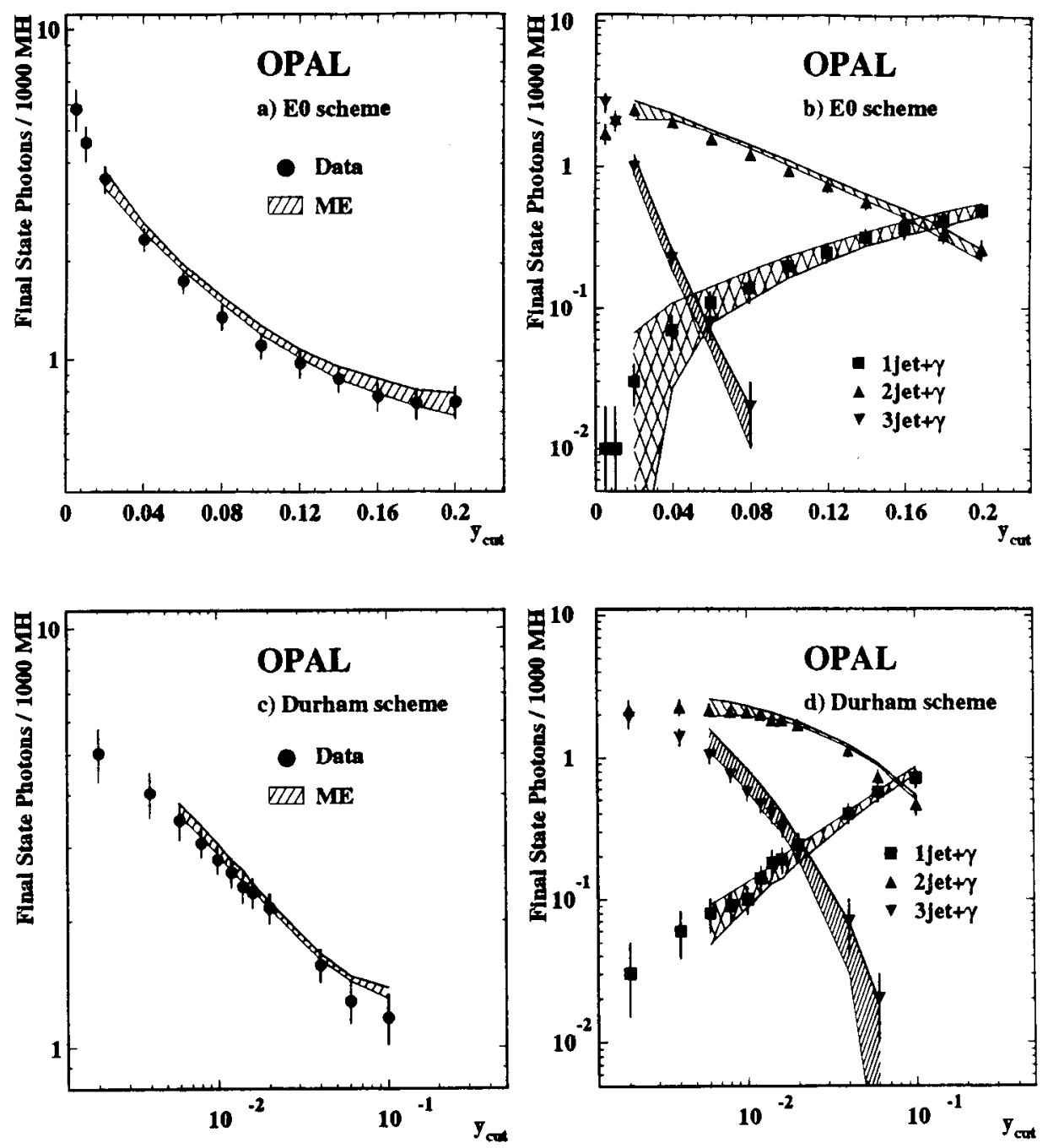

Fig. 1 a-d. Number of events with final state photons per 1000 multihadronic events (points with error bars) compared to the prediction of the QCD matrix element calculation of [7] (hashed band). The width of the theoretical prediction is due to a variation of $\alpha_{\mathrm{s}}$ and the theoretical photon isolation criterion (see text). a total rate in $E 0$ recombination scheme, $\mathbf{b}$ rate of photon and 1,2 , and 3 jets in $E 0$ scheme, c total rate in Durham recombination scheme, $d$ rate of photon and 1,2 , and 3 jets in Durham scheme. Note that the results for the various $y_{\text {cut }}$ values are correlated and that the results with the Durham scheme contain only the 1991 data

struction as correlated. The others were assumed to be uncorrelated. All error contributions to the combined data sample are listed in Table 4 for three different values of $y_{\text {cut }}$.

\section{The matrix element calculations}

Since our previous publications, Monte Carlo simulations based on an $\mathscr{O}\left(\alpha \alpha_{s}\right)$ matrix element calculation have been developed by Kramer and Spiesberger [7], Glover and Stirling [8], and Kunszt and Trocsanyi [9], to which we shall refer in the following as KS, GS and $\mathrm{KT}$, respectively. For this letter only the KS and GS programs were available to us; for the KT one we use results given to us by its authors [25]. The matrix element Monte Carlos have the advantage over the analytical results of [6], which were used in our previous publications, of providing the four momenta of the quark, antiquark, photon and of the gluon. They allow a formal application of the same analysis method to the partons as to the measured particles of the data, in particular it is possible to perform the jet finding without the photon on the parton level.
Experimentally the partons are not directly observable, therefore the global parton structure first is inferred from the abundant hadrons excluding the photon by clustering them into jets as described above. The photon is considered only in a second step. Because the calculations are of limited order in $\alpha_{s}$, this event definition is not infrared safe, which is particularly relevant for events with a photon and one jet. For example a singular contribution comes from collinear photon emission. At the Born level this may lead to a soft quark $q$ and a hard photon recoiling against a high energy antiquark $\bar{q}$. Since at first the photon is excluded from the jet finding, the quark pair may be merged into a single jet if $y_{\text {cut }}$ is sufficiently large that $y_{q \vec{q}}<y_{\text {cut }}$. This may be true even though $y_{q \gamma} \ll y_{q \bar{q}}$. As a result $y_{\gamma \text { jet }} \sim 1$ and such an event will be retained in the simulated sample irrespective of the value of $y_{g \gamma}$. These events appear as one jet recoiling against a hard photon. A reliable theoretical prediction is therefore only possible within some phase space boundaries excluding the singular region for $y_{q \gamma}=0$ in the quark photon system. Such boundaries arise naturally since gluon emission should dominate over photon emission if the invariant masses in the parton cascade are of the order of the hadron masses and hadronisation effects should 
Table 2. The corrected number of hadronic events that have final state photons per 1000 multihadronic $Z^{0}$ decays for various values of $y_{\text {cut }}$ and the $E 0$ and Durham scheme. The first error combines the statistical and systematic uncertainty of $c_{2}$, added in quadrature, the second is due to the energy and isolation cut taken from the JETSET model. Also shown are the predictions from the matrix element Monte Carlo of [7] and the parton shower models JETSET [12], ARIADNE [13], and HERWIG [14]. The uncertainties in the matrix element calculation are due to a variation of $\alpha_{\mathrm{s}}$ between 0.15 and 0.21 and to the theoretical photon cut-off. The uncertainties assigned to the QCD shower model predictions are only due to variations of the QCD scale parameter $\Lambda$ given by the parameter optimisation [24]. Where no error is quoted, the uncertainties are below 0.005. For the Durham scheme only the 1991 data are used

\begin{tabular}{|c|c|c|c|c|c|}
\hline$y_{\text {cut }}$ & Data & Matrix element & JETSET & ARIADNE & HERWIG \\
\hline \multicolumn{6}{|c|}{$E 0$ scheme } \\
\hline $\begin{array}{l}0.005 \\
0.010 \\
0.020 \\
0.040 \\
0.060 \\
0.080 \\
0.100 \\
0.120 \\
0.140 \\
0.160 \\
0.180 \\
0.200\end{array}$ & $\begin{array}{l}5.81 \pm 0.35 \pm 0.76 \\
4.59 \pm 0.27 \pm 0.50 \\
3.57 \pm 0.20 \pm 0.29 \\
2.33 \pm 0.15 \pm 0.12 \\
1.74 \pm 0.12 \pm 0.09 \\
1.35 \pm 0.10 \pm 0.07 \\
1.11 \pm 0.08 \pm 0.06 \\
0.98 \pm 0.08 \pm 0.05 \\
0.88 \pm 0.07 \pm 0.04 \\
0.78 \pm 0.07 \pm 0.04 \\
0.74 \pm 0.07 \pm 0.04 \\
0.75 \pm 0.07 \pm 0.05\end{array}$ & $\begin{array}{l}3.53 \pm 0.20 \pm 0.10 \\
2.53 \pm 0.07 \pm 0.07 \\
1.92 \pm 0.02 \pm 0.06 \\
1.53 \pm 0.02 \pm 0.05 \\
1.24 \pm 0.01 \pm 0.04 \\
1.05 \pm 0.01 \pm 0.03 \\
0.92 \pm 0.02 \pm 0.03 \\
0.84 \pm 0.02 \pm 0.03 \\
0.77 \pm 0.03 \pm 0.03 \\
0.74 \pm 0.03 \pm 0.03\end{array}$ & $\begin{array}{l}4.67 \pm 0.07 \\
3.76 \pm 0.06 \\
2.85 \pm 0.06 \\
1.99 \pm 0.04 \\
1.53 \pm 0.03 \\
1.19 \pm 0.03 \\
0.97 \pm 0.02 \\
0.82 \pm 0.01 \\
0.70 \pm 0.01 \\
0.62 \pm 0.01 \\
0.57 \pm 0.01 \\
0.54 \pm 0.01\end{array}$ & $\begin{array}{l}6.15 \pm 0.03 \\
4.88 \pm 0.02 \\
3.67 \pm 0.01 \\
2.58 \pm 0.01 \\
1.98 \pm 0.01 \\
1.57 \pm 0.01 \\
1.29 \pm 0.01 \\
1.11 \\
1.00 \\
0.90 \\
0.83 \\
0.80\end{array}$ & $\begin{array}{l}6.08 \pm 0.04 \\
4.88 \pm 0.03 \\
3.64 \pm 0.03 \\
2.48 \pm 0.02 \\
1.87 \pm 0.02 \\
1.47 \pm 0.02 \\
1.19 \pm 0.02 \\
1.01 \pm 0.01 \\
0.86 \pm 0.01 \\
0.75 \pm 0.01 \\
0.68 \pm 0.01 \\
0.64 \pm 0.01\end{array}$ \\
\hline \multicolumn{6}{|c|}{ Durham scheme } \\
\hline
\end{tabular}

become important. In the following we discuss the implementation of the phase space boundaries and their impact on the prediction of the different Monte Carlos, from which we estimate their theoretical uncertainties.

Since the calculations predict at most three jets that accompany the photon, we restrict our analysis to a region where the fraction of events with more than three jets in the data is small, i.e. we consider only 0.02 $<y_{\text {cut }}<0.2$ (E0 scheme) and $0.006<y_{\text {cut }}<0.1$ (Durham scheme) for the comparison with the matrix element calculations.

The KS Monte Carlo bounds the integration region in terms of the scaled invariant mass $M_{i j}^{2} / E_{\mathrm{cm}}^{2}$. Two minimum masses are assumed. The variable $y_{0}$ defines a lower limit in the $q \bar{q} g \gamma$ phase space: for $y_{q g}<y_{0}$ events do not contain a resolved $q g$ pair but contribute to the $q \bar{q} \gamma$ final states. Secondly, the photon isolation parameter $y_{\gamma}$ is used to exclude events with small masses of the photon and either of the two quarks. To avoid negative contributions of the sum of virtual and singular real corrections to the $q \bar{q} \gamma$ cross section, the authors recommend $y_{\gamma}$ values greater than $0.5 \cdot 10^{-3}[26]$. The upper bound has to be chosen so as to avoid cutting into the phase space of the $y_{\text {cut }}$ used by experiment, i.e. $y_{y}<y_{\text {cut }}$. We consider only $y_{y} \leqq 5 \cdot 10^{-3}$. Fixing $y_{y}$ at $10^{-3}$ but varying $y_{0}$ between $10^{-5}$ and $10^{-7}$ we observe stable solutions for the jet rates for the physical $y_{\text {cut }}$ between 0.02 and 0.2 . On the other hand, fixing $y_{0}$ at $10^{-6}$ and varying $y_{\gamma}$ between $0.5 \cdot 10^{-3}$ and $5 \cdot 10^{-3}$, we observe small variations of $\leqq 5 \%$ for $y_{\text {cut }}$ between 0.04 and 0.16 ( $E 0$ scheme). For the following results we therefore use $y_{0}=10^{-6}$ and $y_{\gamma}=10^{-3}$. We interpret the variation of the results with the photon isolation parameter in the range $0.5 \cdot 10^{-3}$ to $5 \cdot 10^{-3}$ as theoretical uncertainty.

The GS and KT Monte Carlo calculations are based on another set of variables to define the phase space limits. In addition to $y_{0}$ they introduce a minimum photon energy $E_{\min }$ and a minimum isolation angle $\theta_{\min }$ of the photon with respect to any parton. These variables are related to the experimental $y_{\text {cut }}$ through $y_{\text {cut }}>2 E_{\text {jet }} E_{\text {min }}\left(1-\cos \theta_{\text {min }}\right)$.

To soft gluons, which are experimentally not resolvable, the isolation requirement is not applied to ensure their divergent contributions cancel against virtual corrections. For example in the GS calculation, gluons with an energy of less than about $E_{\min } \cdot y_{0} / y_{\text {cut }}$, i.e. about 200 to $300 \mathrm{MeV}$, are allowed inside the cone around the photon. Glover and Stirling find stable solutions for a $y_{0}$ value of about $1 \%$ of the experimental $y_{\text {cut }}$. Both calculations use as default values $E_{\min }=7.5 \mathrm{GeV}$ and $\theta_{\min }=15$ degrees. Note, however, that in the theoretical calculation the isolation cut is used with respect to at most three 
partons whereas the experiment applies the isolation cut with respect to typically 30 hadrons. Due to the energy cut in the calculations of Glover and Stirling and of Kunszt and Trocsanyi we expect that for low values of $y_{\text {cut }}$ our corrected cross section exceeds their predictions. We restrict our comparison to these models to values of $y_{\text {cut }} \geqq 0.06$ in the $E 0$ scheme. At $y_{\text {cut }}=0.06$ the expected difference to the energy cut in the simulation is $\sim 2.5 \%$ and the prediction has been corrected accordingly. For higher values of $y_{\text {cut }}$ this difference vanishes.

Before comparing the matrix element calculations with the data we want to discuss how the corrections $c_{1}$, described in the previous section, that link the hadronic final state with the partons provided by the predictions, depend on the phase space for photon emission. These corrections are required since, firstly, in the experiment cuts have to be imposed on the energy and the isolation of the photon candidate, and secondly, the much larger multiplicity of hadrons over partons prohibits a direct comparison of data and theoretical calculation. The losses due to the cuts and the quality of the jet reconstruction are determined using QCD shower models as described in Sect. 4. In principle this procedure depends on the way the singular contributions close to the phase space boundaries are treated, or correspondingly, how the cut-off for photon emission is implemented. The cut-offs in the matrix element calculation are different from those used in the QCD shower models. In the latter these are defined by a parameter $Q_{\gamma}$ that terminates the evolution of the parton showering into photons and is treated differently in the various models. JETSET defines a minimum virtual quark mass $m_{q}$, ARIADNE uses a minimum transverse momentum, while HERWIG uses approximately $E_{i} \sqrt{1-\cos \theta_{j k}}$ for a branching $i \rightarrow j+k$. A corresponding cut-off, $Q_{g}$, exists for the gluon emission and as a default the models assume $Q_{\gamma}=Q_{\mathrm{g}} \sim 1 \mathrm{GeV}$. The value of $Q_{\gamma}$ cannot be calculated theoretically, but since it parametrises the boundary between parton showering and hadronisation it should be of the order of hadron masses. We note that the recommended cut-off for the KS Monte Carlo also fulfils this condition: $y_{y}$ corresponds to masses of $2-6 \mathrm{GeV}$.

Since no unambiguous connection between QCD shower models and matrix element calculation can be made, we study how the correction $c_{1}$ depends on the choice of the cut-off parameters used in the QCD shower models. As discussed in the last section, the correction factors obtained with the JETSET, ARIADNE and HERWIG models agree within 5\% at least for $y_{\text {cut }}$ values below 0.16 . As an additional check we varied $Q_{\gamma}$, between $10 \mathrm{MeV}$ and $5 \mathrm{GeV}$ within the shower models. Although the number of generated photons depends strongly on $Q_{\gamma}$, the correction is largely independent of it. Changes of the correction factors were only significant under two conditions. Empirically we find that the corrections, adopting the E0 scheme, increase if $Q_{\gamma}>$ $0.3 \sqrt{y_{\text {cut }}} E_{\mathrm{cm}}$, where phase space effects become important. This corresponds to a $Q_{y} \sim 2 \mathrm{GeV}$ at $y_{\text {cut }}=0.005$ or $\sim 5 \mathrm{GeV}$ at $y_{\text {cut }}=0.04$. In addition we find the correction for the one jet rate increases by $\sim 5 \%$ at large $y_{\text {cut }}$ values for an increase of $Q_{y}$ from 2 to $5 \mathrm{GeV}$. Under all other conditions we observe no statistically significant variation (i.e. not larger than $2 \%$ ). From these studies we conclude that neither the value of the cut-off for photon emission nor the detailed definition of the cut-off procedure in the shower models have significant effects on the correction factor $c_{1}$.

These considerations lead us to believe that we can make meaningful comparison of our data with the matrix element calculations.

\section{Photon rates and the matrix element calculations}

The matrix element calculations have two sets of free parameters: the strong coupling constant $\alpha_{\mathrm{s}}$, which affects both the total rate of photon events and the relative proportions of one, two and three jet events, and the electroweak coupling constants, which affect the total rate only. This means that the parameters can be obtained from the data separately. One can obtain $\alpha_{s}$ from the jet rates, and using this value the electroweak quark coupling constants can be determined.

The total rate of events with final state photons as a function of $y_{\text {cut }}=M_{i j}^{2} / E_{\text {vis }}^{2}$ is shown in Fig. 1 and listed in Table 2. As discussed before we adopt the $E 0$ and the Durham recombination schemes. Note that only the 1991 data are used for the Durham scheme. We observe a fast decrease of the photon rate with increasing $y_{\text {cut }}$, which in the $E 0$ scheme tends to level off at $y_{\text {cut }} \sim 0.18$. In Fig. $1 \mathrm{~b}$, $\mathrm{d}$ and Table 3 we display for the two recombination schemes the absolute rate of events with $n$ jets and a photon, for $n=1,2$, and 3 . For both recombination schemes the rate of three or more jets dominates at very low values of $y_{\text {cut }}$, but falls steeply with $y_{\text {cut }}$. The two jet rate exhibits a maximum around $y_{\text {cut }}=0.02$ ( $E 0$ scheme) and 0.004 (Durham scheme). The one jet rate increases continuously with $y_{\text {cut }}$ and becomes dominant around $y_{\text {cut }}=0.17$ ( $E 0$ scheme) and 0.09 (Durham scheme). For a $y_{\text {cut }}$ value of 0.02 in the $E 0$ scheme the relative jet rates are about the same as at 0.01 in the Durham scheme, but the total photon yield is about $30 \%$ larger in the $E 0$ scheme. As the following results will show, this difference does not affect any of the conclusions.

As discussed above the comparison of two and three jet cross sections in the data and the calculation can be used to determine $\alpha_{s}$. Note that the calculation is only of first order and therefore has no explicit dependence on the renormalisation scale. The ratio

$$
R_{3}^{\gamma}=\frac{\sigma_{3 \mathrm{jet}+\gamma}}{\sigma_{2 \mathrm{jet}+\gamma}+\sigma_{3 \mathrm{jet}+\gamma}}
$$

is a measure of the first order $\alpha_{\mathrm{s}}$ value, $\alpha_{\mathrm{s}}^{(1)}$, relevant for this analysis and is independent of the total cross section. In the $E 0$ scheme we compare the observed ratio $R_{3}^{\gamma}$ with the matrix element Monte Carlo of Kramer and Spiesberger [7] for various values of $\alpha_{s}^{(1)}$. We do this at the two $y_{\text {cut }}$ values of 0.02 and 0.04 , where the rate of larger than three jets is small and the sensitivity to $\alpha_{s}^{(1)}$ is still sizeable. The values are summarised in Table 5 and are in agreement with the $\alpha_{\mathrm{s}}$ value in first order obtained 
Table 3. Number of events per 1000 multihadronic $Z^{0}$ decays with a photon and 1,2,3 or more jets for different values of $y_{\text {cur }}$. The first error combines the statistical and systematic uncertainty of $c_{2}$, added in quadrature, the second is due to the energy and isolation cut. If no error is quoted, the uncertainties are below 0.005. For the Durham scheme only the 1991 data are used

\begin{tabular}{|c|c|c|c|c|}
\hline$y_{\text {cut }}$ & 1 jet & 2 jet & 3 jet & $>3$ jet \\
\hline \multicolumn{5}{|c|}{ E0 scheme } \\
\hline $\begin{array}{l}0.005 \\
0.010 \\
0.020 \\
0.040 \\
0.060 \\
0.080 \\
0.100 \\
0.120 \\
0.140 \\
0.160 \\
0.180 \\
0.200\end{array}$ & $\begin{array}{l}0.01 \pm 0.01 \\
0.01 \pm 0.01 \\
0.03 \pm 0.01 \\
0.07 \pm 0.02 \pm 0.01 \\
0.11 \pm 0.02 \pm 0.01 \\
0.14 \pm 0.03 \pm 0.01 \\
0.20 \pm 0.03 \pm 0.01 \\
0.25 \pm 0.04 \pm 0.01 \\
0.32 \pm 0.04 \pm 0.02 \\
0.36 \pm 0.05 \pm 0.02 \\
0.41 \pm 0.05 \pm 0.02 \\
0.49 \pm 0.05 \pm 0.03\end{array}$ & $\begin{array}{l}1.68 \pm 0.17 \pm 0.22 \\
2.14 \pm 0.16 \pm 0.24 \\
2.47 \pm 0.16 \pm 0.20 \\
2.03 \pm 0.13 \pm 0.10 \\
1.55 \pm 0.11 \pm 0.08 \\
1.20 \pm 0.09 \pm 0.06 \\
0.92 \pm 0.07 \pm 0.05 \\
0.73 \pm 0.07 \pm 0.04 \\
0.56 \pm 0.06 \pm 0.03 \\
0.42 \pm 0.05 \pm 0.02 \\
0.33 \pm 0.04 \pm 0.02 \\
0.26 \pm 0.04 \pm 0.02\end{array}$ & $\begin{array}{l}2.77 \pm 0.21 \pm 0.36 \\
2.02 \pm 0.15 \pm 0.22 \\
0.99 \pm 0.09 \pm 0.08 \\
0.23 \pm 0.04 \pm 0.01 \\
0.08 \pm 0.02 \\
0.02 \pm 0.01\end{array}$ & $\begin{array}{l}1.30 \pm 0.12 \pm 0.17 \\
0.37 \pm 0.05 \pm 0.04 \\
0.06 \pm 0.02\end{array}$ \\
\hline \multicolumn{5}{|c|}{ Durham scheme } \\
\hline
\end{tabular}

Table 4. Relative error contributions in $\%$ to the total final state photon cross section of the combined data sample from 1990 and 1991 for three different values of $y_{\text {cut }}$ in the $E 0$ scheme. The row labelled 'Sum' contains the sum of the first six errors, added in quadrature and is quoted as the first error in the text and in Tables 2 and 3

\begin{tabular}{lccc}
\hline Source of error & \multicolumn{3}{c}{ Relative Errors in \% for $E 0$ scheme } \\
\cline { 2 - 4 } & $y_{\text {cut }}=0.005$ & $y_{\text {cut }}=0.06$ & $y_{\text {cut }}=0.20$ \\
\hline Statistics & 3.7 & 4.9 & 7.6 \\
Syst. error of jet energies & 0.5 & 1.3 & 1.4 \\
Monte Carlo statistics & 2.2 & 2.6 & 3.9 \\
Background & 4.1 & 3.4 & 3.2 \\
Acceptance of multihadrons & 0.4 & 0.4 & 0.4 \\
Photon efficiency & 1.0 & 1.0 & 1.0 \\
\hline Sum & 6.0 & 6.7 & 9.3 \\
\hline Energy and isolation cut & 13.0 & 5.0 & 7.0 \\
\hline
\end{tabular}

from the jet rates in inclusive multihadronic decays of the $Z^{0}$ [28]. In the Durham scheme similar values of $R_{3}^{v}$ are observed at $y_{\text {cut }}$ values of 0.01 and 0.02 . The corresponding values of $\alpha_{s}$ can also be found in Table 5. For this recombination scheme the values are lower than the $\alpha_{\mathrm{s}}^{(1)}$ obtained from a fit to the jet rates in inclusive multihadrons [28].

After fixing $\alpha_{s}$ from the ratio of two and three jet events, we now compare the theoretical prediction of the absolute rates with our data over the range $0.02<y_{\text {cut }}<0.20$, assuming standard model values for the electroweak coupling constants. To take into account
Table 5. First order $\alpha_{\mathrm{s}}$ values for different values of $y_{\text {cut }}$ in the $E 0$ and Durham scheme as obtained by the Kramer and Spiesberger Monte Carlo for the measured fraction of three jet plus photon events $R_{3}^{\gamma}$. The first order $\alpha_{\mathrm{s}}$ value obtained from the jet rates in inclusive hadronic $Z^{0}$ decays is also listed for both recombination schemes

\begin{tabular}{lll}
\hline$y_{\text {cut }}$ & $R_{3}^{\gamma}$ & $\alpha_{\mathrm{s}}^{(1)}$ \\
\hline$E 0$ scheme & & \\
0.02 & $0.288 \pm 0.019$ & $0.176 \pm 0.010$ \\
0.04 & $0.104 \pm 0.015$ & $0.197 \pm 0.026$ \\
Inclusive multihadrons & & $0.177 \pm 0.013$ \\
Durham scheme & & \\
0.01 & $0.230 \pm 0.011$ & $0.168 \pm 0.008$ \\
0.02 & $0.121 \pm 0.007$ & $0.186 \pm 0.011$ \\
Inclusive multihadrons & & $0.21 \pm 0.01$ \\
\hline
\end{tabular}

the spread of the $\alpha_{\mathrm{s}}$ values for various $y_{\text {cut }}$ values and from the inclusive multihadronic events, we use $\alpha_{\mathrm{s}}^{(1)}$ $=0.18 \pm 0.03$ for both schemes. This range of $\alpha_{\mathrm{s}}$ includes all of the $\alpha_{\mathrm{s}}$ values quoted above and amounts to about three standard deviations of the individual measurements. The corresponding KS predictions are shown in Table 2 and by the shaded bands in Fig. 1. The widths of these bands are due to the $\alpha_{\mathrm{s}}$ range and the variation of the theoretical photon isolation parameter. The variation of the total photon rate with $\alpha_{\mathrm{s}}$ for the range stated above, is about $6 \%$ for $y_{\text {cut }}$ values around 0.02 and 0.2 but drops to about $1 \%$ for intermediate values of $y_{\text {cut }}$. In 

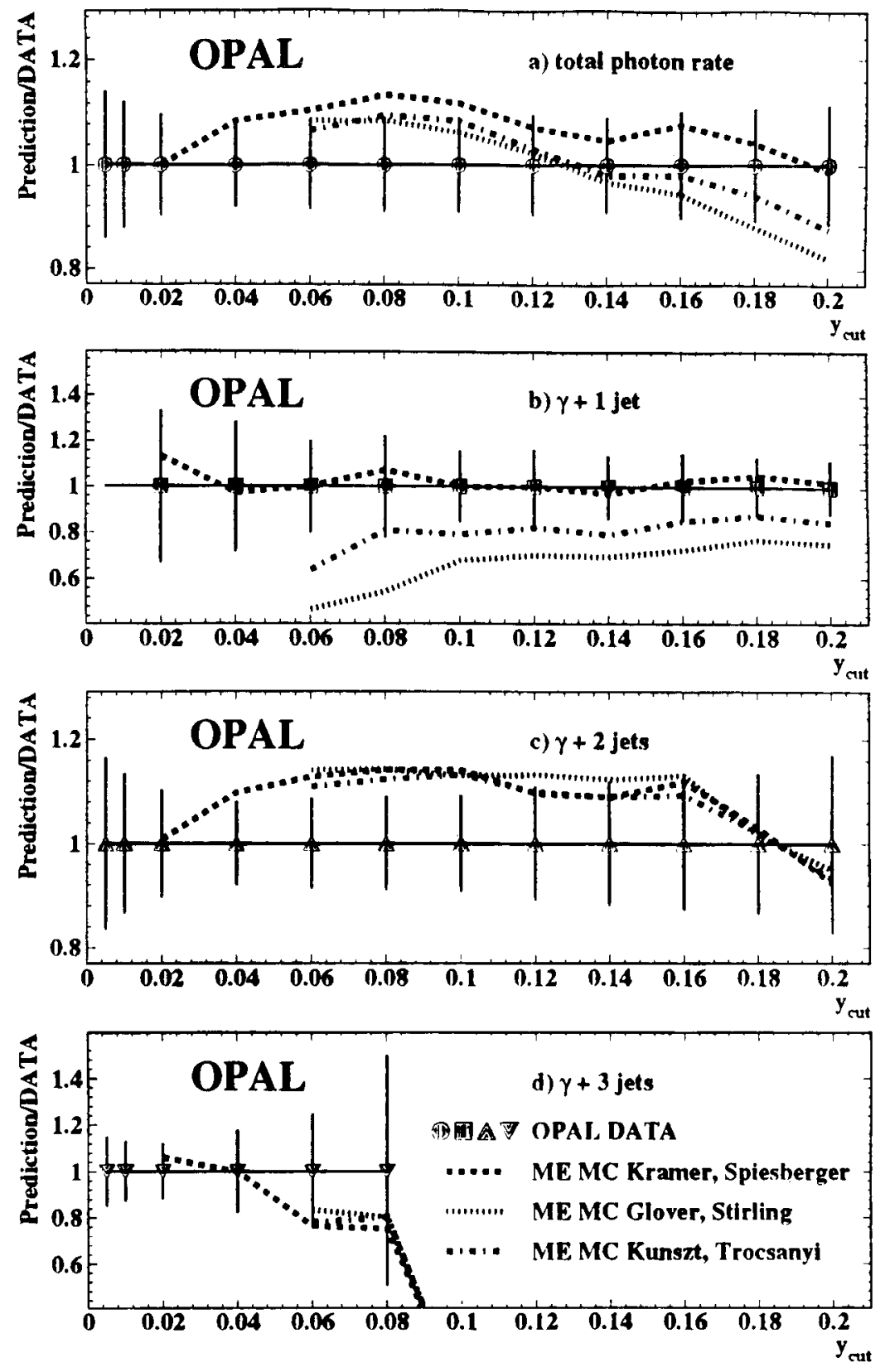

Fig. 2a-d. Ratio of the prediction of matrix element calculations over data as a function of $y_{\text {cut }}$ in the $E 0$ scheme. Shown is the ratio for $\mathbf{a}$ the total rate, $\mathbf{b}$ the photon plus one jet rate, $\mathbf{c}$ the photon plus two jet rate, and $\mathbf{d}$ the photon plus three jet rate. The error bars on the measurement give the relative experimental error. The lines denote the theoretical predictions of Kramer and Spiesberger (dashed), Glover and Stirling (dotted) and Kunszt and Trocsanyi (dashed-dotted). In each case a value of $\alpha_{\mathrm{s}}=\mathbf{0 . 1 8}$ was assumed. Note that an uncertainty in the theoretical calculation of typically $5 \%$ has to be taken into account general we observe a good agreement in the total rate between the calculation and the data for both recombination schemes and over the entire $y_{\text {cut }}$ range considered. This can also be seen in Fig. 2 a where we plot the ratio of prediction over measurement for $\alpha_{\mathrm{s}}^{(1)}=0.18$. The error bars indicate the relative error of the data. Note that an uncertainty in the theoretical calculation of typically $5 \%$, due to the variation of the theoretical photon isolation parameter and due to the uncertainty in $\alpha_{s}$, has to be taken into account. For $0.06<y_{\text {cut }}<0.1$ the calculation is somewhat above the data. However, the significance is only about 1.5 standard deviations.

The prediction for the jet rates is displayed in Figs. $1 \mathrm{~b}$ and $d$ and $2 b-d$. Note that the three jet rate increases but the one jet rate decreases with increasing $\alpha_{s}$. As a function of $\alpha_{s}$, the two jet rate decreases at low $y_{\text {cut }}$ but increases at large values. These partial cancellations imply that the dependence of the total cross section on $\alpha_{s}$ is much smaller than that for individual jet rates. The prediction is in general in agreement with the data for both schemes. For values of $y_{\text {cut }}$ between 0.04 and 0.16 in the $E 0$ scheme the prediction for the two jet rate is about $10 \%$ above the data. The significance is, however, only about 1.5 standard deviations.

As can be seen from the bands in Figs. 1b, d, and from Fig. 2, the one jet rate is also well described by the matrix element calculation of Kramer and Spiesberger [7]. In our previous publications [3,4] we observed that the Kramer Lampe calculation [6] underestimates the measured one jet rate by a factor of four to five, which we assigned to the different definitions of a photon event. Using the matrix element Monte Carlo from Kramer and Spiesber- 

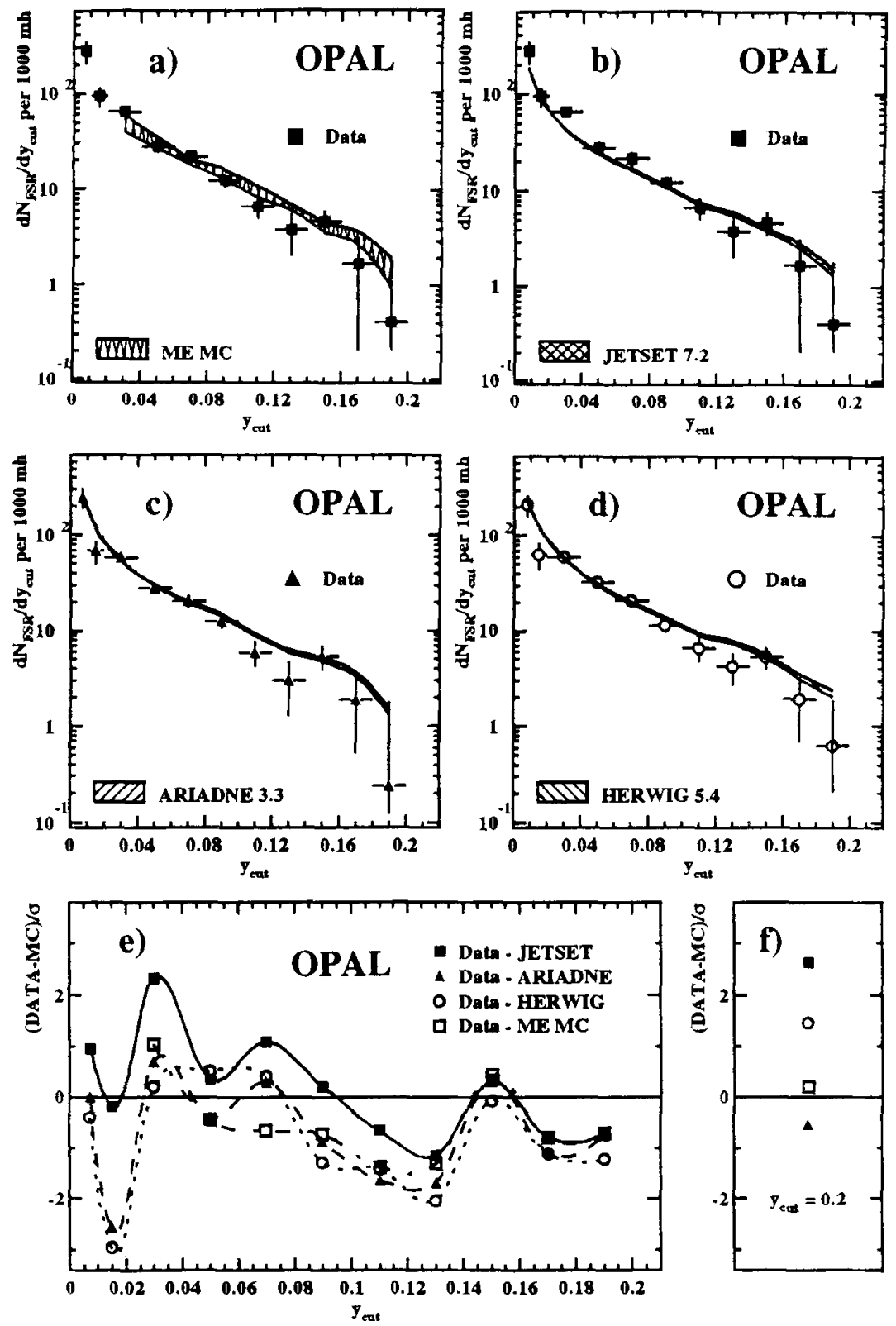

Fig. 3a-f. Differential rate $D_{\mathrm{FSR}}\left(y_{\text {cut }}\right)$ compared to the predictions of a the matrix element calculation [7], b JETSET, c ARIADNE and d HERWIG. The data in a and $b$ are corrected using JETSET, in $c$ and $\mathrm{d}$ using the respective models. e Difference of the theoretical prediction and the data in terms of standard deviations for the four theoretical calculations. f The difference of $N_{\mathrm{FSR}}$ between theory and data in terms of standard deviations for $y_{\text {cut }}=0.2$ ger the discrepancies vanish. The dependence on $\alpha_{\mathrm{s}}$ is significantly larger for the one jet rate than for the other jet rates. It is about $40 \%$ at low $y_{\text {cut }}$ and about $14 \%$ at large $y_{\text {cut }}$ for a change of $\alpha_{\mathrm{s}}$ between 0.15 and 0.21 . This sensitivity to $\alpha_{\mathrm{s}}$ can be understood if one refers to the example of the one jet event with hard photon and a soft quark discussed in Sect. 4. Additional gluon emission increases the invariant mass of the hadronic part of the event and consequently the chance of splitting it into two jets, of which one might fail the condition $y_{\gamma, \text { jet }}>y_{\text {cut }}$ leading to a rejection of the event. This $\alpha_{\mathrm{s}}$ dependence may be related to the singularity due to collinear photon emission, discussed in the previous section [9].

Since the data of Table 2 and Fig. 1 are correlated for different values of $y_{\text {cut }}$, it is difficult to quantify the quality of the theoretical prediction. Better variables for this purpose are the differential distributions $D_{\mathrm{FSR}}$, described in Sect. 4, which statistically are almost inde- pendent for the different values of $y_{\text {cut }}$. The result is shown in Fig. 3a.

We define a $\chi^{2}$ for the measurement and theoretical prediction in the $E 0$ scheme

$\chi^{2}=\Delta^{T} V^{-1} \Delta$.

With the column vector

$\Delta=\left(\begin{array}{c}D_{\mathrm{FSR}}^{\text {meas. }}\left(y_{\text {cut }}\right)_{1}-D_{\mathrm{FSR}}^{\text {model }}\left(y_{\text {cut }}\right)_{1} \\ \cdots \\ \cdots \\ D_{\mathrm{FSR}}^{\text {meas. }}\left(y_{\text {cut }}\right)_{n}-D_{\mathrm{FSR}}^{\text {model }}\left(y_{\text {cut }}\right)_{n} \\ N_{\mathrm{FSR}}^{\text {meas. }}(0.2)-N_{\mathrm{FSR}}^{\text {model }}(0.2)\end{array}\right)$

containing the differences of the measured and predicted differential rates $D_{\text {FSR }}$ for all $n y_{\text {cut }}$ bins. The last component of $\Delta$ contains the difference of the measured and 
simulated final state photon rate $N_{\mathrm{FSR}}$ at the highest value of $y_{\text {cut }}$ to account for a possible offset of the two differential distributions.

The covariance matrix, $V$, contains in its off-diagonal elements $V_{i j}=\delta^{2} N_{\mathrm{FSR}}\left(y_{\text {cut }}\right)_{i} N_{\mathrm{FSR}}\left(y_{\text {cut }}\right)_{j}$ the common experimental uncertainties affecting all intervals of $y_{\text {cut }}$ with $\delta$ denoting the sum in quadrature of all correlated relative errors. These uncertainties are due to the photon efficiency, the jet energy reconstruction and the energy and isolation requirement on the photon, and part of the background estimate. These contributions are estimated as $\delta=0.031$. In addition the uncertainty in $\alpha_{\mathrm{s}}$ and the variation of the theoretical photon cut-off, which also affects all $y_{\text {cut }}$ values in a similar way, was taken into account. This leads to the total correlated relative uncertainty of $\delta=0.047$. The diagonal elements $V_{i i}$ are the quadratic sum of the statistical error, the systematic error specific to the particular $y_{\text {cut }}$ and the total correlated error common to all bins of $y_{\text {cut }}$, as discussed above.

We observe a good agreement between data and the KS matrix element calculaion. We find $\chi^{2} /$ d.o.f. $=0.80$ for 10 degrees of freedom. This prediction can accommodate the measurement for all values of $y_{\text {cut }}$.

We also compare our data with the calculation of Glover and Stirling [8] described in Sect. 4. As for the calculation of Kramer and Spiesberger we determine the effective $\alpha_{\mathrm{s}}$ in the $E 0$ scheme from $R_{3}^{\gamma}$ at $y_{\text {cut }}=0.04$. Given the measured value of $R_{3}^{\gamma}=0.104 \pm 0.015$ (see above), we find a value of $\alpha_{s}^{(1)}=0.182 \pm 0.025$, which is in good agreement with the value of $0.197 \pm 0.026$ of the $\mathrm{KS}$ calculation. The requirement of a minimum photon energy of $7.5 \mathrm{GeV}$ in the GS Monte Carlo potentially affects the two and three jet cross sections differently, leading to a bias in the $\alpha_{\mathrm{s}}$ determination. We study this effect using the JETSET model and find it to be less than the statistical uncertainty of $R_{3}^{\gamma}$. For the comparison of the data with the GS and KT calculations $[8,9]$ we use $\alpha_{s}=0.18$. Calculating the jet rates with the program of Glover and Stirling we find good agreement with the data and between the two matrix element calculations for the two and three jet rates for $y_{\text {cut }} \geq 0.06$. The same applies to the calculation of Kunszt and Trocsanyi [25] for this value of $\alpha_{\mathrm{s}}$. We also compare in Fig. 2 the results of these two calculations with our data for $y_{\text {cut }} \geq 0.06$. To correct for the expected losses at $y_{\text {cut }}=0.06$ due to the $E_{\min }$ cut used in the GS and KT calculations, we increase their prediction by $2.5 \%$. For $y_{\text {cut }}<0.06$ the photon energy cut reduces the phase space involved in the two calculations and as a consequence their predicted photon rates fall below the data. The agreement with the data is good with the possible exception of the one jet rate that is systematically below the data but still compatible within the errors: $10-20 \%$ in the calculation of KT and $25-50 \%$ in the calculation of GS possibly indicating a non-perturbative contribution [9].

As can be seen from Fig. 2, the calculations agree with each other for the two and three jet rate within $3 \%$. Larger discrepancies are observed only in the one jet rate. We therefore conclude that at least in the region where the one jet cross section is not high, the calculations are in good agreement with each other.

\section{Comparison with QCD shower models}

We compare our data, corrected to the parton level, with the three available QCD shower models that include photon emission. We use the models with parameters optimised to describe the global event topologies of inclusive multihadronic $Z^{0}$ decays $[24,15]$. Such a comparison is a test of the modelling of the parton evolution and is largely independent of the hadronisation prescription. Since our previous publications photon emission has been included in HERWIG. After modifications of the model to allow for large angle photon and gluon emission the authors found that it gives a good description of the published data [15]. Our results together with the model predictions are given in Table 2.

To quantify the consistency of the models with the data we compare the model prediction for the differential distribution $D_{\mathrm{FSR}}\left(y_{\mathrm{cut}}\right)$ with the measurement. Related to the above discussion about the model dependence of the differential correction factors we correct our measurement separately for the different models. The results are shown in Fig. 3b-d. The differences in terms of standard deviations are shown in Fig. 3e. We observe the following results for 12 degrees of freedom. As discussed above, the correlated relative error $\delta$ was 0.031 with an additional model specific contribution from the uncertainty due to the $A_{\mathrm{QCD}}$ value which amounted to $0.015,0.005$ and 0.010 for JETSET, ARIADNE and HERWIG, respectively. JETSET has a $\chi^{2} /$ d.o.f. $=1.81$ corresponding to a confidence level (C.L.) of 0.041 . The shape is general well reproduced, but at the offset at $y_{\text {cut }}=0.2$ underestimates the data by about $2.5 \sigma$. ARIADNE has a $\chi^{2} /$ d.o.f. $=1.35($ C.L. $=0.185)$. HERWIG has a $\chi^{2} /$ d.o.f. $=1.84($ C.L. $=0.037)$. At the current level of accuracy ARIADNE and to a lesser extent HERWIG and JETSET can reproduce the measured differential photon cross section $D_{\text {FSR }}\left(y_{\text {cut }}\right)$.

\section{Electroweak quark couplings}

The matrix element calculations have two sets of physical parameters; the strong coupling strength $\alpha_{s}$ and the electroweak quark couplings. The value of $\alpha_{s}$ has been fixed from the relative jet rates leaving the electroweak couplings as the only free parameters which may influence the absolute photon yield. Based on the KS matrix element calculation we now translate our measurement into the values of the electroweak couplings of quarks. The uncertainties of the prediction originate from the theoretical photon cut-off, the variation in $\alpha_{\mathrm{s}}^{(1)}$ and the hadronisation correction.

As suggested in [10], the measurement of the photon yield together with the hadronic width of the $Z^{0}$ allows a determination of the electroweak couplings of up and down type quarks. The measured quantities can be expressed in terms of the coupling constants $c_{i}=v_{i}^{2}+a_{i}^{2}$ $(i=u, d)$ as follows. The partial width into photons is

$\Gamma\left(Z^{0} \rightarrow \gamma+\right.$ jets $)\left(y_{\text {cut }}\right)=\frac{h}{9} \frac{\alpha}{2 \pi} F\left(y_{\text {cut }}\right)\left[8 c_{u}+3 c_{d}\right]$, 
and the total hadronic width is

$$
\begin{aligned}
& \Gamma\left(Z^{0} \rightarrow q \bar{q}\right) \\
& \quad=h\left(1+\frac{\alpha_{\mathrm{s}}^{(2)}}{\pi}+1.41\left(\frac{\alpha_{\mathrm{s}}^{(2)}}{\pi}\right)^{2}\right)\left[2 c_{u}+3 c_{d}\right],
\end{aligned}
$$

where

$h=3 \frac{G_{F} M_{Z 0}^{3}}{24 \pi \sqrt{2}}$.

Here $F\left(y_{\text {cut }}\right)$ is the theoretical KS prediction [7], discussed above, for the fraction of photon events retained for some $y_{\text {cut }}$ and with $\alpha_{\mathrm{s}}^{(1)}=0.18, G_{F}$ is the Fermi coupling constant at the muon mass, $M_{Z^{0}}$ the mass of the $Z^{0}, a_{\mathrm{s}}^{(2)}=0.122+0.006$ [28] is the strong coupling constant in second order and $\alpha$ is the electromagnetic coupling constant, $a_{i}$ and $v_{i}$ denote the axial and vector couplings defined by $v_{i}=2 I_{3, i}^{L}-4 \cdot Q_{i} \sin ^{2} \theta_{W}$ and $a_{i}=2 I_{3, i}$. Here $I_{3}^{L}, Q$, and $\theta_{W}$ are the third component of the weak isospin, the charge of the quarks and the weak mixing angle, respectively.

The measurement of the photon rate at $y_{\text {cut }}=0.06$ in the $E 0$ scheme, where the theoretical and experimental errors are smallest, gives the combination

$8 c_{u}+3 c_{d}=12.36 \pm 0.78 \pm 0.64 \pm 0.29$,

where the first error is due to statistics and experimental systematics, the second error comes from the energy and isolation requirement, and the third one from the uncertainty in $\alpha_{s}$ and the theoretical cut-off. Assuming the electroweak couplings of all up type and of all down type quarks to be the same, which is expected to be the case within $2-3 \%$, we combine this results with $\Gamma\left(Z^{0} \rightarrow\right.$ hadrons $)=1.740 \pm 0.012 \mathrm{GeV}[29]$. The allowed regions for the two combinations of up and down type quark couplings are shown in Fig. $4 \mathrm{a}$. We solve these combinations for $c_{u}$ and $c_{d}$ and obtain

$c_{u}=0.94 \pm 0.13 \pm 0.11 \pm 0.05$;

$c_{d}=1.62 \pm 0.09 \pm 0.07 \pm 0.03$ or

$\Gamma_{u}=242 \pm 34 \pm 28 \pm 12 \mathrm{MeV} ;$

$\Gamma_{d}=419 \pm 23 \pm 19 \pm 7 \mathrm{MeV}$.

Similarly, in the Durham scheme the theoretical and experimental uncertainties are smallest for $y_{\text {cut }}=0.014$, giving $\Gamma_{u}=261 \pm 49 \mathrm{MeV}$, and $\Gamma_{d}=406 \pm 29 \mathrm{MeV}$, here all error contributions are added in quadrature. These results are consistent with those from the $E 0$ scheme. This agreement suggests that potential theoretical uncertainties in comparing the theoretical calculation for massless partons and massive jets are small.

The errors on these results are more than twice as small as those in our previous publication. The main improvement is due to the higher statistics and the better theoretical foundation; the total theoretical uncertainty of the previous analysis was dominated by the different event definitions used in the data and the theoretical calculation and led to an estimated error of almost $10 \%$. For this analysis only the value of $\alpha_{\mathrm{s}}^{(1)}$ and the photon cut-off is relevant, leading to a theoretical uncertainty of about $3 \%$ of the electroweak coupling constants.

The partial widths from this analysis can be compared with those of the $Z^{0}$ into charm and bottom quarks using various methods. As shown in Fig. 4b, the results of this analysis are in good agreement with the combined measurements from all LEP experiments using various methods [30]. The errors from this analysis and of the combined LEP values are similar. All these measurements are in agreement with the expectation from the standard model of $\left(\Gamma_{u}+\Gamma_{c}\right) / 2=296.0 \mathrm{MeV}$ and $\left(\Gamma_{d}+\Gamma_{s}+\Gamma_{b}\right) / 3$ $=380.3 \mathrm{MeV}$ [31] assuming a top mass of $130 \mathrm{GeV} / \mathrm{c}^{2}$, a Higgs mass of $300 \mathrm{GeV} / \mathrm{c}^{2}$ and $\alpha_{\mathrm{s}}^{(2)}=0.12$.

\section{Summary}

Based on about 1000 observed multihadronic $Z^{0}$ decays with final state photons we performed a comparison of the data with recent theoretical calculations. We observe that a Monte Carlo for a matrix element calculation of $\mathscr{O}\left(\alpha \alpha_{\mathrm{s}}\right)$ gives a good account of the data. The value of
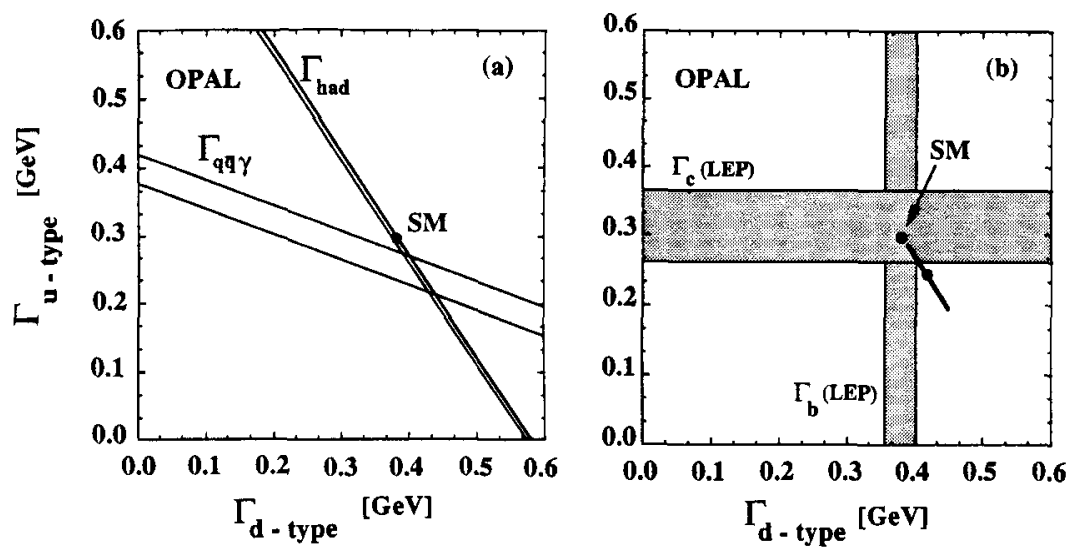

Fig. 4a, b. a Correlation of the decay widths of the $Z^{0}$ into up and down type quarks, $\Gamma_{u}$ versus $\Gamma_{d}$. Shown are the relations obtained within one standard deviation from the measured hadronic width and from the photon yield compared to the prediction of the matrix element calculation [7]. Also indicated is the standard model expectation. b Correlation plot of $\Gamma_{u}$ versus $\Gamma_{d}$ as obtained from this measurement. Displayed is the one standard deviation contour (narrow bar). Also shown are the partial widths of the $Z^{0}$ into charm and bottom quarks, combined from all LEP experiments. The standard model value for the average of up and charm, and down, strange and bottom quarks, respectively, is indicated 
$\alpha_{\mathrm{s}}^{(1)}$ required to describe the jet rates in photon events is in agreement with the value obtained from inclusive multihadronic $Z^{0}$ decays. Now that identical procedures for event definition can be applied to theoretical calculations and data, the discrepancy in the one jet rate, which was apparent in our previous publications, vanishes or is at least considerably reduced. Final state photon emission is also a probe of the parton evolution as modelled in QCD shower simulations. We find a confidence level of 0.19 for ARIADNE, and of 0.04 for HERWIG and JETSET. The reduced theoretical errors and the increased statistics lead to a significantly improved measurement of the electroweak couplings of up and down type quarks. The partial decay widths of the $Z^{0}$ into up type quarks is $\Gamma_{u}=242 \pm 46 \mathrm{MeV}$ and into down type quarks $\Gamma_{d}=419 \pm 30 \mathrm{MeV}$. Their precision is similar to the results for charm and bottom quarks obtained from various methods and by combining results from all LEP experiments.

Acknowledgements. For this analysis we profited from numerous discussions with N. Glover, Z. Kunszt, M. Seymour, H. Spiesberger, W.J. Stirling, and Z. Trocsanyi. Their help and advice is gratefully acknowledged. It is a pleasure to thank the SL Division for the efficient operation of the LEP accelerator, the precise information on the absolute energy, and their continuing close cooperation with our experimental group. The computer centres at CERN and RAL have provided excellent facilities for the data handling and analysis. In addition to the support staff at our own institutions we are pleased to acknowledge the Department of Energy, USA, National Science Foundation, USA, Science and Engineering Research Council, UK, Natural Sciences and Engineering Research Council, Canada, Israeli Ministry of Science, Minerva Gesellschaft, Japanese Ministry of Education, Science and Culture (the Monbusho) and a grant under the Monbusho International Science Research Program, American Israeli Bi-national Science Foundation, Direction des Sciences de la Matière du Commissariat à l'Energie Atomique, France, Bundesministerium für Forschung und Technologie, FRG, National Research Council of Canada, Canada, A.P. Sloan Foundation and Junta Nacional de Investigação Científica e Tecnológica, Portugal.

\section{References}

1. T.F. Walsh, P. Zerwas: Phys. Lett. 44B (1973) 195; S.J. Brodsky, C.E. Carlson, R. Suaya: Phys. Rev. D14 (1976) 2264; K. Koller, T.F. Walsh, P. Zerwas: Z. Phys. C2 (1979) 197; E. Laermann, T.F. Walsh, I. Schmitt, P.M. Zerwas: Nucl. Phys. B207 (1982) 205

2. OPAL Coll., M.Z. Akraway et al.: Phys. Lett. B246 (1990) 285

3. OPAL Coll., G. Alexander et al.: Phys. Lett. B264 (1991) 219

4. OPAL Coll., P.D. Acton: Z. Phys. C54 (1992) 193
5. ALEPH Coll., D. Decamp et al.: Phys. Lett. B264 (1991) 476; DELPHI Coll., P. Abreu et al.: Z. Phys. C53 (1992) 555; L3 Coll., O. Adriani et al.: Phys. Lett. B292 (1992) 472; ALEPH Coll., D. Buskulic et al.: CERN PPE/92-143

6. G. Kramer, B. Lampe: Phys. Lett. B269 (1991) 401

7. G. Kramer, H. Spiesberger : in: the Proceedings of the Workshop on Photon Radiation from Quarks, Annecy, 1991, S. Cartwright (ed.), CERN 92-04; DESY 92-022

8. E.W.N. Glover, J. Stirling: DTP $92-52$

9. Z. Kunszt, Z. Trocsanyi: ETH-TH/92-26

10. P. Mättig, W. Zeuner: Z. Phys. C52 (1991) 31

11. See contributions in: Proceedings of the Workshop on Photon Radiation from Quarks, Annecy, 1991, S. Cartwright (ed.), CERN 92-04

12. T. Sjöstrand; Comput. Phys. Commun. 39 (1986), 347; JETSET, Version 7.2

13. U. Petterson: LU TP 88-5 (1988); U. Petterson, L. Lönnblad: LU TP 88-15 (1988); L. Lönnblad: LU TP 89-10 (1989); L. Lönnblad: in: Proceedings of the Workshop on Photon Radiation from Quarks, Annecy, 1991, S. Cartwright (ed.), CERN 92-04

14. G. Marchesini, B.R. Webber: Nucl. Phys. B238 (1984) 1; B.R. Webber: Nucl. Phys. B238 (1984) 492; G. Abbiendi et al.: Comput. Phys. Commun. 67 (1992) 465

15. M.H. Seymor: in: Proceedings of the Workshop on Photon Radiation from Quarks, Annecy, 1991, S. Cartwright (ed.), CERN 92-04; Cavendish-HEP 91/16

16. OPAL Coll., K. Ahmet et al.: Nucl. Instrum. Methods A305 (1991) 275

17. OPAL Coll., G. Alexander et al.: Z. Phys. C52 (1991) 175

18. JADE Coll., W. Bartel et al.: Z. Phys. C33 (1986) 23; JADE Coll., S. Bethke et al.: Phys. Lett. B 123 (1988) 235

19. $\mathrm{Yu}$. Dokshitzer: contribution to the Workshop on Jets at LEP and HERA, Durham 1990; N. Brown, W.J. Stirling: Z. Phys. C53 (1992) 629; S. Catani et al.: Phys. Lett. B269 (1991) 432; S. Bethke, Z. Kunszt, D.E. Soper, W.J. Stirling: Nucl. Phys. B370 (1992) 310

20. OPAL Coll., M.Z. Akrawy et al.: Phys. Lett. B253 (1991), 511

21. F.A. Berends, R. Kleiss, S. Jadach: Nucl. Phys. B202 (1982) 63

22. R. Brun et al.: GEANT3 User's Guide, CERN DD/EE/84-1 (1989); J. Allison et al.: Nucl. Instrum. Methods A 317 (1992) 47

23. P.P. Allport et al.: submitted to Nucl. Instrum. Methods A

24. OPAL Coll., M.Z. Akrawy et al.: Z. Phys. C47 (1990) 505

25. Z. Kunszt, Z. Trocsanyi: private communication

26. H. Spiesberger: private communication

27. Private communication: Y. Dokshitzer, R. Kleiss, P.M. Zerwas; B. Kniehl: in: Proceedings of the Workshop on Photon Radiation from Quarks, Annecy, 1991, S. Cartwright (ed.), CERN 92-04

28. OPAL Coll., P.D. Acton et al.: Z. Phys. C55 (1992) 1

29. The LEP Colls.: Phys. Lett. B276 (1992) 247

30. The combination of LEP measurements was taken from: Particle Data Group, K. Hikoso et al.: Phys. Rev. D45 (1992)

31. D. Bardin et al.: CERN-TH-6443-92 